\title{
Oesophageal Cancer: Conformal Radiotherapy vs. Hybrid-VMAT Technique With Two Different Treatment Planning Systems
}

\author{
MASAYOSHI MIYAZAKI ${ }^{1,2}$, SHINGO OHIRA ${ }^{2,3}$, YOSHIHIRO UEDA ${ }^{2,3}$, MASARU ISONO $^{2}$, \\ MASAYUKI FUJIWARA ${ }^{1}$, MASAO TANOOKA ${ }^{1}$, WATARU OKADA ${ }^{1}$, RYUTA NAKAHARA ${ }^{1}$, \\ MASAKI SUEOKA ${ }^{1}$, HITOMI SUZUKI ${ }^{1}$, TERUKI TESHIMA ${ }^{2}$ and KOICHIRO YAMAKADO ${ }^{1}$ \\ ${ }^{1}$ Department of Radiology, Hyogo College of Medicine, Hyogo, Japan; \\ ${ }^{2}$ Department of Radiation Oncology, Osaka International Cancer Institute, Osaka, Japan; \\ ${ }^{3}$ Department of Radiation Oncology, Osaka University Graduate School of Medicine, Suita, Japan
}

\begin{abstract}
Background/Aim: Traditionally, the radiotherapy of oesophageal cancer has been conformal radiotherapy $(C R T)$. We sought to compare dosimetric parameters of conformal radiotherapy (CRT) with those of two treatment planning systems for hybrid-volumetric modulated arc therapy (h-VMAT) for the treatment of oesophageal cancer. Patients and Methods: In 11 patients, we compared: i) planning target volume coverage, ii) dose to organs at risk, and iii) the dose rate (DR) of the three techniques. We evaluated two treatment planning systems: i) Eclipse and ii) RayStation. Results: The Conformity Index of the CRT plan was significantly higher for the h-VMAT plans, compared to all other parameters. Normal lung tissue volumes receiving $>5$, 13, or 20 Gy were lower with the RayStation plan compared to Eclipse. The volume of cardiac tissue receiving $>40$ Gy was highest with the CRT plan. The minimum DR in VMAT was lowest for the RayStation plan (49.5 MU/min). Conclusion: The h-VMAT plan using RayStation is the appropriate choice for reducing lung dose.
\end{abstract}

Oesophageal cancer is a common cause of cancer death around the world (1). Chemoradiotherapy for oesophageal cancer has better local control and overall survival compared to chemotherapy (2). A cisplatin-based combination is the

This article is freely accessible online.

Correspondence to: Masayoshi Miyazaki, Department of Radiology, Hyogo College of Medicine, 1-1, Mukogawa-cho, Nishinomiya, Hyogo, 663-8501, Japan. Tel: +81 669451181, Fax: +81 669451900, e-mail: masayoshi.miyazaki@gmail.com

Key Words: Hybrid-VMAT, treatment planning, plan evaluation, oesophageal cancer. standard regimen of chemoradiotherapy for oesophageal cancer (3).

The radiotherapy (RT) technique for oesophageal cancer has commonly been conformal radiation therapy (CRT). This technique is often limited by the dose to the organs at risk (OARs). The National Cancer Institute (NCI) does not permit intensity-modulated radiotherapy (IMRT) for treatments in the thorax of patients in NCI-sponsored trials $(4,5)$. IMRT generally produces widely distributed but lower doses of radiation to normal tissues surrounding the planning target volume (PTV) compared to CRT. The normal tissue volumes involved during RT for oesophageal cancer are larger compared to those in lung cancer treatment because of the longer cranio-caudal length of the PTV.

Volumetric modulated arc therapy (VMAT) has recently been used for the treatment of oesophageal cancer (6). To reduce the volume of normal tissue that receives these extraneous doses, Mayo et al., (7) have developed a new technique called 'Hybrid-IMRT'. This technique combines static and IMRT beams used concurrently, and has produced results with a better dose conformity and sparing of OARs using VMAT with a shorter treatment time compared to IMRT (8). For this reason, we used volumetric modulated arc therapy (VMAT) instead of IMRT (9). Multiple commercial treatment planning systems (TPSs) have become available. Lafond et al., (10) have compared the dosimetric parameters of two VMAT treatment planning systems for prostate cancer, whereas Langner et al., (11) have compared point doses between Eclipse and Raystation (Eclipse Version 13.7.29; Varian Medical Systems, Palo Alto, CA, USA and RayStation version 4.7.4.4, RaySearch Medical Laboratories AB, Stockholm, Sweden) for proton therapy. The result of calculation is difficult between TPSs, because the calculation algorithm was different. Comparison of hybrid-VMAT (h-VMAT) techniques using two treatment planning systems has not been done before. 
The aim of this study was to compare dosimetric parameters of CRT with those of two h-VMAT plans from two different treatment planning systems for the treatment of oesophageal cancer.

\section{Patients and Methods}

Patients. Subjects were 11 cases of oesophageal cancer patients treated with h-VMAT technique at our institute between 2017 and 2018. Patient characteristics are summarized in Table I. This study was approved by our ethics committee (No. 42718).

Treatment planning. For the computed tomography (CT) simulation, patients were immobilized with a vacuum pillow (Vac-Lock, Civco Medical Solutions, Iowa, USA) in the supine position with the arms raised. CT images were acquired using the Revolution HD (GE Medical Systems, Milwaukee, WI, USA). The parameters for CT acquisitions were: i) $2 \mathrm{~mm}$ slices, ii) $512 \times 512$ matrix, and iii) 50 $\mathrm{cm}$ field of view. These images were transferred to the Eclipse TPS (Eclipse Version 13.7.29; Varian Medical Systems).

The CRT plan used only Eclipse. The h-VMAT plans used both Eclipse and RayStation. The dose calculation used the analytical anisotropic algorithm (AAA), while the h-VMAT plan from RayStation was recalculated by the AAA of Eclipse.

Target volumes and OARs (e.g., the lung, heart, and spinal cord) were contoured by radiation oncologists. The gross tumour volume (GTV) consisted of identified masses and clinical target volumes (CTV) for boosting and was created by adding potential volumes of tumour extension to GTV. CTV for elective treatment included lymph node regions at potential risk of occult metastasis. The PTV was created by adding an isotropic margin of $5 \mathrm{~mm}$ to the CTV. In all cases, high-risk planning target volume (PTVboost) was based on the primary and clinical lymph node metastases, while PTVelective included elective dose areas.

The dose prescriptions were 40 and 20 Gy for the PTVelective and PTVboost (2 Gy/fraction), respectively. For the CRT plan, doses were delivered to the isocentre. For the h-VMAT plans, doses were prescribed to the mean dose to the PTV. The CRT plan consisted of opposite anterior-posterior (AP) fields to deliver PTVelective and opposite oblique fields to deliver PTVboost to the isocentre. The h-VMAT plans used two full arcs in VMAT and opposite AP fields. The prescription dose of the h-VMAT plan was delivered at 50\% with VMAT, and $50 \%$ in the AP direction. The following dose constraints were used for OARs: i) maximal dose (Dmax) to the spinal cord at $\leq 45 \mathrm{~Gy}$, ii) $V_{5}$ for the lung at $\leq 65 \%$, and iii) $V_{20}$ for the lung at $\leq 35 \%$. While maintaining dose constraints of PTV coverage and spinal cord, the three plans had added constraints to minimize lung dose.

The typical field arrangements and isodose distribution from 5 Gy to max dose of the three treatment techniques are illustrated in Figure 1.

The following dosimetric parameters were analysed: i) $\mathrm{D}_{98}$, ii) $\mathrm{D}_{95}$, iii) $\mathrm{D}_{2}$, iv) conformity index (CI) for PTV, v) homogeneity index (HI) for PTV, vi) Dmax to the spinal cord, vii) $V_{5}$, viii) $V_{13}$, ix) $\left.V_{20}, x\right) V_{30}$ and $x i$ ) mean of lung-PTVelective (MLD), xii) $V_{40}$ and xiii) mean to heart. Dx\% indicates the dose that includes $x \%$ of the target and $\mathrm{Vx} \%$ indicates the volume $\%$ of the target receiving $\mathrm{x} \%$ of the prescribed dose. CI was determined by dividing the volume receiving the prescribed dose by the target volume. HI was evaluated as the difference between $\mathrm{D}_{2}$ and $\mathrm{D}_{98}$ of PTV divided by
Table I. Patient characteristics.

\begin{tabular}{llc}
\hline & & Patients (n=11) \\
\hline \multirow{2}{*}{ Age } & Median & 66 \\
& Range & $38-89$ \\
Gender & Male & 9 \\
Histology & Female & 2 \\
Primary site & Squamous cell carcinoma & 11 \\
& Ut & 3 \\
& Ut-Mt & 3 \\
& Mt & 2 \\
Stage & Mt-Lt & 1 \\
& Lt & 2 \\
& I & 2 \\
& II & 1 \\
& III & 4 \\
PTVelective Volume (cc) & IVA & 2 \\
& Median & 2 \\
PTVboost Volume (cc) & Range & Median \\
& Range & 532.5 \\
PTVelective CCL (cm) & Median & $284.8-912.1$ \\
& Range & 225.7 \\
PTVboost CCL (cm) & Median & $105.6-328.4$ \\
& Range & 23.9 \\
& & $13.4-31.2$ \\
& & 15.82 \\
& & $9-24.4$ \\
\hline
\end{tabular}

Ut: Upper thoracic; Mt: middle thoracic; Lt: lower thoracic. PTV: planning target volume; CCL: craniocaudal length.

$\mathrm{D}_{50}$ of PTV. The Dx of PTVboost was calculated adding plans of PTVelective and PTVboost. The dose rates (DR:MU/minute) of the two arc beams of two h-VMAT plans were compared.

Data analysis. Results are expressed as mean \pm standard deviation. Paired Student's $t$-tests were used to compare the dosimetric parameters. A value of $p<0.017$ was defined as having statistical significance using the Bonferroni's method.

\section{Results}

Target coverage. Table II shows the dosimetric parameters of PTV for CRT and the two h-VMAT plans. $\mathrm{D}_{95}$ of PTVelective and PTVboost were not significantly different and $>95 \%$ of the prescribed dose. The $\mathrm{D}_{50}$ of PTVelective for the Eclipse plan was not significantly different from that of the RayStation plan. The $\mathrm{D}_{50}$ for PTVboost of the Eclipse plan was significantly different from that of the RayStation plan $(p<0.01)$. The CI for PTVelective of the CRT plan was significantly higher compared to that of the Eclipse and RayStation plans $(p<0.001)$. The CI for PTVboost of the Eclipse plan was also significantly lower compared to that of the RayStation plan $(p<0.001)$.

Organs at risk. Table II shows OAR doses. The $\mathrm{V}_{5}$ for lung-PTVelective of the Eclipse plan was significantly 

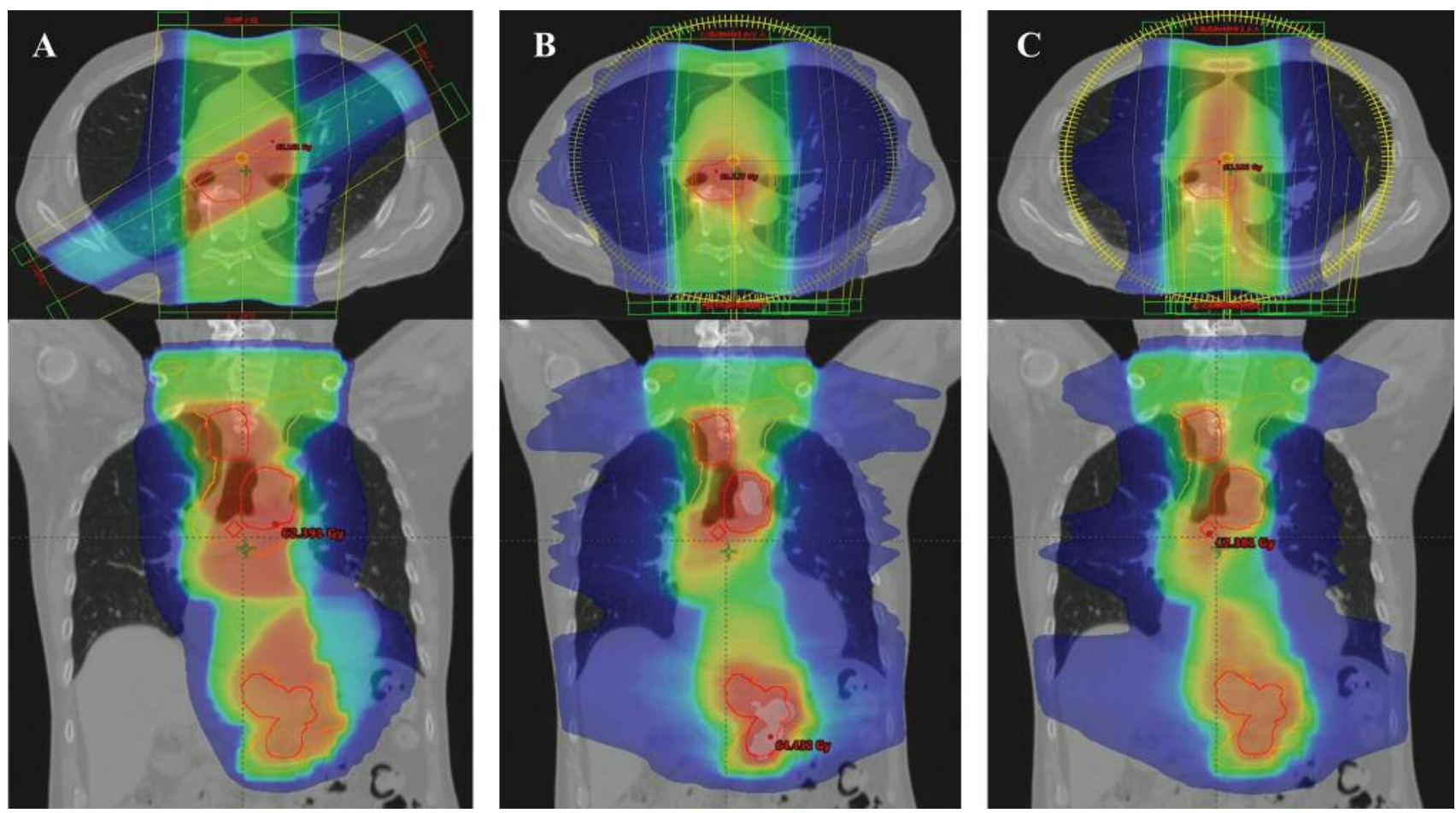

Figure 1. Axial (upper) and coronal (lower) dose distribution of $>5$ Gy for the three planning techniques for one patient. (A) CRT plan, (B) h-VMAT plan using Eclipse, (C) h-VMAT plan using RayStation. The color of dose distribution is $>5$ Gy (blue), $>20$ Gy (light blue), >30 Gy (green), >45 Gy (yellow), >55 Gy (orange).

higher compared to those of the CRT and RayStation plans $\left(p<0.001\right.$ and 0.01 , respectively). The $\mathrm{V}_{13}$ for lungPTVelective of the RayStation plan was significantly lower compared to that of the CRT plan $(p=0.005)$. The $\mathrm{V}_{20}$ for lung-PTVelective of the RayStation plan was significantly lower compared to that of the CRT plan $(p<0.001)$. The $\mathrm{V}_{30}$ for lung-PTVelective of the CRT plan was not significantly different from those of the Eclipse and RayStation plans. The mean dose for lung-PTVelective of the CRT plan was significantly different from those of the Eclipse and RayStation plans. The maximum spinal cord dose of the CRT plan was significantly higher than that of the Eclipse and RayStation plans $(p=0.026$ and 0.035 , respectively).

Dose rate. The mean DR of the RayStation plan was significantly higher compared to that of the Eclipse plan $(p<0.001)$. The minimum DR of the RayStation plan was lower compared to that of the Eclipse plan $(49.53 \mathrm{MU} / \mathrm{min} v s$. $62.06 \mathrm{MU} / \mathrm{min})$. The beams of the CRT plan and AP beams of the h-VMAT plan were $600 \mathrm{MU} / \mathrm{min}$ stably. Figure 2 shows an example of DR for h-VMAT plans between Eclipse and RayStation.

\section{Discussion}

In this study, we compared the dosimetric parameters of CRT and of two h-VMAT plans using two treatment planning systems in 11 oesophageal cancer patients. The two h-VMAT plans showed a significant improvement in CI compared to CRT. The h-VMAT plan using RayStation in CI showed good coverage compared to Eclipse plans. In normal lung, the RayStation plan provided lower low-dose volumes except for the $\mathrm{V}_{5}$ of the CRT plan. The heart $\mathrm{V}_{40}$ was highest in CRT compared the other plans. The Dmax to spinal cord was not significantly different among all methods. The minimum of DR was lowest for the RayStation plan (49.5 MU/min), though the RayStation plan had a wider range of DR compared to the Eclipse plan.

Previous publications on IMRT/VMAT have recognized some advantages compared to CRT (12-14). However, the IMRT/VMAT plans give a higher lung dose compared to the CRT plans (V5, V10, V20, MLD) (15). To reduce the volume of normal lung for V5, V13, V20, V30, MLD, the h-VMAT plan offered better conformity and lower low-dose volumes except for the $V_{5}$ of the lung (9). It is generally understood that $\mathrm{V}_{20}$ and mean lung dose (MLD) are major predictors for 
Table II. Dose parameters of PTV coverage, OARs.

\begin{tabular}{|c|c|c|c|c|c|c|}
\hline & \multirow[b]{2}{*}{ CRT } & \multirow[b]{2}{*}{$\mathrm{EC}$} & \multirow[b]{2}{*}{ RS } & \multicolumn{3}{|c|}{$p$-Value } \\
\hline & & & & CRT vs. EC & CRT vs. RS & EC $v s . \mathrm{RS}$ \\
\hline \multicolumn{7}{|l|}{ PTVel } \\
\hline D98 (\%) & $36.61 \pm 0.70$ & $36.96 \pm 0.56$ & $36.39 \pm 0.98$ & 0.11 & 0.56 & 0.11 \\
\hline D95 (\%) & $37.41 \pm 0.62$ & $37.75 \pm 0.48$ & $37.74 \pm 0.48$ & 0.16 & 0.21 & 0.94 \\
\hline D50 (\%) & $39.98 \pm 0.62$ & $40.15 \pm 0.14$ & $40.26 \pm 0.11$ & 0.39 & 0.15 & 0.04 \\
\hline D2 (\%) & $42.14 \pm 0.88$ & $41.92 \pm 0.65$ & $41.55 \pm 0.33$ & 0.52 & 0.05 & 0.11 \\
\hline \multicolumn{7}{|l|}{ PTVb } \\
\hline D98 (\%) & $54.54 \pm 3.65$ & $56.61 \pm 1.05$ & $56.33 \pm 1.54$ & 0.09 & 0.15 & 0.62 \\
\hline D95 (\%) & $56.63 \pm 2.00$ & $57.59 \pm 0.85$ & $57.65 \pm 1.04$ & 0.85 & 0.76 & 0.89 \\
\hline D50 (\%) & $60.39 \pm 1.50$ & $60.32 \pm 0.17$ & $60.56 \pm 0.21$ & 0.87 & 0.71 & $<0.01$ \\
\hline $\mathrm{D} 2(\%)$ & $62.04 \pm 1.63$ & $62.45 \pm 0.82$ & $61.95 \pm 0.54$ & 0.74 & 0.88 & 0.38 \\
\hline \multicolumn{7}{|l|}{ CI } \\
\hline PTVel & $2.65 \pm 1.14$ & $0.58 \pm 0.07$ & $0.7 \pm 0.12$ & $<0.001$ & $<0.001$ & $<0.001$ \\
\hline PTVb & $1.42 \pm 1.50$ & $0.66 \pm 0.07$ & $0.83 \pm 0.13$ & 0.11 & 0.2 & $<0.001$ \\
\hline \multicolumn{7}{|l|}{$\mathrm{HI}$} \\
\hline PTVel & $0.14 \pm 0.02$ & $0.12 \pm 0.03$ & $0.13 \pm 0.03$ & 0.7 & 0.37 & 0.16 \\
\hline PTVb & $0.12 \pm 0.06$ & $0.09 \pm 0.03$ & $0.09 \pm 0.03$ & 0.16 & 0.16 & 0.99 \\
\hline \multicolumn{7}{|l|}{ Lung-PTVel } \\
\hline V5 (\%) & $48.81 \pm 15.40$ & $75.25 \pm 17.61$ & $56.38 \pm 9.88$ & $<0.001$ & 0.19 & $<0.01$ \\
\hline V13 (\%) & $30.68 \pm 11.41$ & $23.09 \pm 6.96$ & $18.76 \pm 5.58$ & 0.07 & 0.005 & 0.12 \\
\hline $\mathrm{V} 20(\%)$ & $23.92 \pm 9.06$ & $12.32 \pm 4.02$ & $11.86 \pm 4.22$ & $<0.001$ & $<0.001$ & 0.8 \\
\hline V30 (\%) & $10.22 \pm 3.97$ & $7.07 \pm 3.28$ & $7.52 \pm 3.39$ & 0.06 & 0.1 & 0.76 \\
\hline Mean (\%) & $29.43 \pm 3.38$ & $10.8 \pm 2.16$ & $9.58 \pm 1.89$ & 0.6 & 0.13 & 0.17 \\
\hline \multicolumn{7}{|l|}{ Heart } \\
\hline V40 (\%) & $40.54 \pm 26.77$ & $22.88 \pm 17.63$ & $29.43 \pm 20.03$ & 0.08 & 0.28 & 0.42 \\
\hline Mean (\%) & $26.77 \pm 11.69$ & $25.24 \pm 9.88$ & $25.93 \pm 9.97$ & 0.74 & 0.86 & 0.87 \\
\hline \multicolumn{7}{|l|}{ Spinal cord } \\
\hline Dmax (Gy) & $44.64 \pm 0.49$ & $43.25 \pm 1.85$ & $43.55 \pm 1.51$ & 0.026 & 0.035 & 0.67 \\
\hline
\end{tabular}

PTV: Planning target volume; Dx: the percentage of the prescribed dose covering x\% volume of PTV; CI: conformity index; HI: homogeneity index; $\mathrm{Vx}$ : the percentage of organ receiving $\geq \mathrm{x}$ Gy; EC: Eclipse; RS: Raystation;

radiation pneumonitis (RP). A critical review of the dosevolume effect in the lung recommends limiting $\mathrm{V}_{20}$ to $\leq 30$ $35 \%$ and MLD to 20-23 Gy (16). Schallenkamp et al., (17) have reported results from a study that examined MLD, $V_{10}$, $\mathrm{V}_{13}, \mathrm{~V}_{15}, \mathrm{~V}_{20}, \mathrm{~V}_{30}$, and effective lung dose for the treatment of a series of 99 lung cancer patients. They concluded that larger volumes of lung exposed to lower doses $\left(\mathrm{V}_{13}\right)$ may be more predictive of complications compared to $\mathrm{V}_{20}$ or $\mathrm{V}_{30} . \mathrm{V}_{5}$ may also be important (18). Wu et al., (19) have compared CRT, IMRT, and VMAT in oesophageal cancer patients, and have demonstrated that all plans were able to meet the prescription and there was no clear distinction on PTV coverage. VMAT can decrease the high dose area but delivers more volume of the low dose area. The h-VMAT plan using RayStation compared to CRT and Eclipse successfully lowered the $\mathrm{V}_{20}$ and mean lung dose, however, the low dose was similar to that of the CRT plan, together with an improvement in CI. Regarding the cardiac dosimetry, the RTOG 0617 clinical trial reported that the heart $V_{5}$ and $V_{30}$ are important predictors of patient survival (20). In this study, the heart $\mathrm{V}_{40}$ was extremely high with the CRT plan. This was due to the presence of oblique beams used to reduce the lung dose. The heart $\mathrm{V}_{40}$ remains significantly associated with OS in the multivariable analysis (21).

CIs to PTVelective were significantly higher for the CRT plan. For this reason, a lung volume receiving $>20 \mathrm{~Gy}$ of the CRT plan was significantly higher than that of the Eclipse and RayStation Plans. Chan et al. have compared the CIs of three treatment techniques: i) CRT, ii) VMAT, and iii) h-VMAT for locally-advanced non-small cell lung cancer. They have described that the CIs of VMAT $(1.13 \pm 0.07)$ and h-VMAT $(1.13 \pm 0.05)$ plans were lower than that of CRT (1.42 \pm 0.17 ) (9). Similarly, in our study, the CIs of PTVelective and PTVboost for the CRT plan were higher compared to the other plans. A wide distribution of low dose to the surrounding normal tissues can be harmful for the patients (18). The differences in DRs for the h-VMAT plan between the two treatment systems have not been compared 


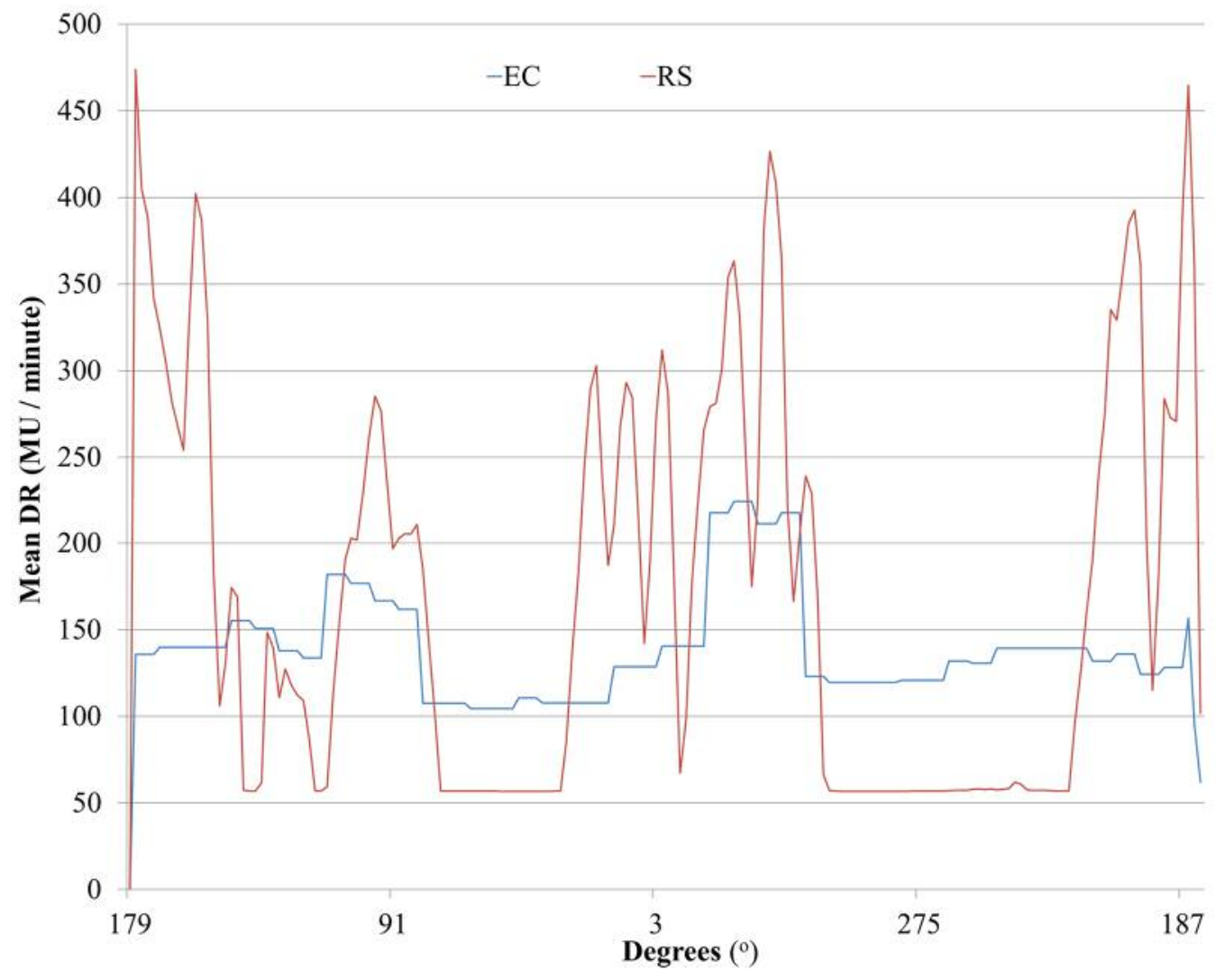

Figure 2. Example of DR for h-VMAT plan between Eclipse (blue) and RayStation (red).

yet. Lafond et al., (10) have compared the DR between two treatment planning systems [Monaco (Elekta, Crawley, UK) and Pinnacle (Philips Medical Systems, Best, the Netherlands)] for VMAT in prostate cancer. The average DR was higher with Monaco (230 MUs/min) compared to Pinnacle (160 MUs/min).

In that study, the VMAT treatment plans obtained with Monaco and Pinnacle offered clinically acceptable dose distributions for prostate cancer (10). The DR of the RayStation plan compared to that of the Eclipse plan was wider and had a lower mean in our study. Due to a wide range of DR, the RayStation plan was a steep distribution compared to the Eclipse plan.

Some limitations exist in this study. First, the $V_{5}$ of lung for the CRT and h-VMAT plan using RayStation were not significantly different. The lung dose of the h-VMAT plan may be reduced by changing the ratio of the A-P beam and VMAT. Second, this study was calculated using AAA. AAA can be suboptimal in low density tissues, such lung, where it may overestimate the dose. Inaccuracy of dose calculation may be reduced by using Monte Carlo (22) or a commercial software (Acuros XB, Varian Medical Systems) (23).
The CI of the h-VMAT plan was significantly lower compared to that of the CRT plan for PTVelective. For this reason, lung doses except for $\mathrm{V}_{5}$ were significantly higher with CRT compared to the h-VMAT plan. The $\mathrm{V}_{5}$ mean of LungPTVelective of the h-VMAT plan using RayStation was significantly lower compared to that of the Eclipse plan. The h-VMAT plan using RayStation is an approach for reducing the lung volume receiving a low dose volume during the treatment of oesophageal cancer.

\section{Conflicts of Interest}

No actual or potential conflicts of interest exist.

\section{Authors' Contributions}

All Authors contributed to data collection, and participated in the writing and final approval of the manuscript.

\section{Acknowledegments}

The Authors would like to thank Libby Cone, MD, MA, from DMC Corp. (www.dmed.co.jp<http://www.dmed.co.jp/>) for editing a draft of this manuscript. 


\section{References}

1 Bray F, Ferlay J, Soerjomataram I, Siegel RL, Torre LA and Jemal A: Global cancer statistics 2018: GLOBOCAN estimates of incidence and mortality worldwide for cancers in 185 countries. CA cancer J Clin 68(6): 394-424, 2085. PMID: 30207593. DOI: $10.3322 /$ caac. 21492

2 Stahl M, Walz MK, Stuschke M, Lehmann N, Meyer HJ, RieraKnorrenschild J, Langer P, Engenhart-Cabllic R, Bitzer M, Königsrainer A, Budach W and Wilke H: Phase III comparison of preoperative chemotherapy compared with chemoradiotherapy in patients with locally advanced adenocarcinoma of the esophagogastric junction. J Clin Oncol 27(6): 851-856, 2009. PMID: 19139439. DOI: 10.1200/JCO.2008.17.0506

3 Nishimura Y, Hiraoka M, Koike R, Nakamatsu K, Itasaka S, Kawamura M, Negoro Y, Araki N, Ishikawa H, Fujii T and Mitsuhashi N: Long-term follow-up of a randomized Phase II study of cisplatine/5-FU concurrent chemoradiotherapy for esophageal cancer (KROSG101/JROSG021). Jpn J Clin Oncol 42(9): 807-812, 2012. PMID: 22811410. DOI: 10.1093/jjco/hys 112

4 Allen AM, Czerminska M, Jänne PA, Sugarbaker DJ, Bueno R, Harris JR, Court L and Baldini EH: Fatal pneumonitis associated with intensity-modulated radiation therapy for mesothelioma. Int J Radiat Oncol Biol Phys 65(3): 640-645, 2006. PMID: 16751058. DOI: 10.1016/j.ijrobp.2006.03.012

5 Tucker SL, Liu HH, Wang S, Wei X, Liao Z, Komaki R, Cox JD and Mohan R: Dose-volume modeling of the risk of postoperative pulmonary complications among esophageal cancer patients treated with concurrent chemotherapy followed by surgery. Int J Radiat Oncol Biol Phys 66(3): 754-761, 2006 PMID: 16965865. DOI: 10.1016/j.ijrobp.2006.06.002

6 Abbas AS, Moseley D, Kassam Z, Kim SM and Cho C: Volumetric-modulated arc therapy for the treatment of a large planning target volume in thoracic esophageal cancer. J Appl Clin Med Phys 14(3): 192-202, 2013. PMID: 23652258. DOI: 10.1120/jacmp.v14i3.4269

7 Mayo CS, Urie MM, Fitzgerald TJ, Ding L, Lo YC and Bogdanov M: Hybrid IMRT for treatment of cancers the lung and esophagus. Int J Radiat Oncol Biol Phys 71(5): 1408-1418, 2008. PMID: 18262730. DOI: 10.1016/j.ijrobp.2007.12.008

8 Vanetti E, Clivio A, Nicolini G, Fogliata A, Ghosh-Laskar S, Agarwal JP, Upreti RR, Budrukkar A, Murthy V, Deshpande DD, Shrivastava SK, Dinshaw KA and Cozzi L: Volumetric modulated arc radiotherapy for carcinomas of the oro-pharynx, hypo-pharynx and larynx a treatment planning comparison with fixed field IMRT. Radiother Oncol 92(1): 111-117, 2009. PMID: 19157609. DOI: 10.1016/j.radonc.2008.12.008

9 Chan OS, Lee MC, Hung AW, Chang AT, Yeung RM and LeeAW: The superiority of hybrid-volumetric arc therapy (VMAT) technique over double arcs VMAT and 3D-conforma technique in the treatment of locally advanced non-small cell lung cancer-a planning study. Radiother Oncol 101(2): 298302, 2011. PMID: 21907438. DOI: 10.1016/j.radonc. 2011.08 .015

10 Lafond C, Gassa F, Odin C, Dréan G, Even J, De Crevoisier R, Pommier P, Manens JP and Biston MC: Comparison between two treatment planning systems for volumetric modulated arc therapy optimization for prostate cancer. Phys Med 30(1): 2-9, 2014. PMID: 23116552. DOI: 10.1016/j.ejmp.2012.10.003
11 Langner UW, Mundis M, Strauss D, Zhu M and Mossahebi S: A comparison of two pencil beam scanning treatment planning systems for proton therapy. J Appl Clin Med Phys 19(1): 156163, 2018. PMID: 29205763. DOI: 10.1002/acm2.12235

12 Liu HH, Wang X, Dong L, Wu Q, Liao Z, Stevens CW, Guerrero TM, Komaki R, Cox JD and Mohan R: Feasibility of sparing lung and other thoracic structures with intensity-modulated radiotherapy for non-small cell lung cancer. Int J Radiat Oncol Biol Phys 58(4): 1268-1279, 2004. PMID: 15001272. DOI: 10.1016/j.ijrobp.2003.09.085

13 Jiang X, Li T, Liu Y, Zhou L, Xu Y, Zhou X and Gong Y: Planning analysis for locally advanced lung cancer: dosimetric and efficiency comparisons between intensity-modulated radiotherapy (IMRT), single-arc/partial-arc volumetric modulated arc therapy (SA/PA-VMAT). Radiother Oncol 6: 140, 2011. PMID: 22014217. DOI: 10.1186/1748-717X-6-140

14 Murshed H, Liu HH, Liao Z, Barker JL, Wang X, Tucker SL, Chandra A, Guerrero T, Stevens C, Chang JY, Jeter M, Cox JD, Komaki R and Mohan R: Dose and volume reduction for normal lung using intensity-modulated radiotherapy for advanced-stage non-small lung cancer. Int J Radiat Oncol Biol Phys 58(4): 1258-1267, 2004. PMID: 15001271. DOI: 10.1016/j.ijrobp.2003. 09.086

15 Rao AS, Amin SS, Narayanan SS and Narayanan GS: Dosimetric comparison of volumetric modulated arc therapy and three-dimensional conformal radiotherapy during post mastectomy left chest wall irradiation. Int J Cancer Res Ther Radiat Oncol Biol Phys 2(2): 1-4, 2017. DOI: 10.33140/IJCRT/02/02/00007

16 Marks LB, Bentzen SM, Deasy J, Kong FM, Bradley JD, Vogelius IS, EI Naga I, Hubbs JL, Lebesque JV, Timmerman RD, Martel MK and Jackson A: Radiation dose-volume effects in the lung. Int J Radiat Oncol Biol Phys 76(3): S70-76, 2010. PMID: 2017521. DOI: 10.1016/j.ijrobp.2009.06.091

17 Schallenkamp JM, Miller RC, Brinkmann DH, Foote T and Garces YI: Incidence of radiation pneumonitis after thoracic irradiation: dose-volume correlates. Int J Radiat Oncol Biol Phys 67(2): 410-416, 2007. PMID: 17236964. DOI: 10.1016/j.ijrobp. 2006.09.030

18 Wang S, Liao Z, Wei X, Liu HH, Tucker SL, Hu CS, Mohan R, Cox JD and Komaki R: Analysis of clinical and dosimetric factors associated with treatment-related pneumonitis (TRP) in patients with non-small-cell lung cancer (NSCLC) treated with concurrent chemotherapy and three-dimensional conformal radiotherapy (3D-CRT). Int J Radiat Oncol Biol Phys 66(5): 1399-1407, 2006. PMID: 16997503. DOI: 10.1016/j,ijrobp.2006. 07.1337

19 Wu Z, Xie C, Hu M, Han C, Yi J, Zhou Y, Yuan H and Jin X: Dosimetric benefits of IMRT and VMAT in the treatment of middle thoracic esophageal cancer: is the conformal radiotherapy still an alternative option? J Appl Clin Med Phys 15(3): 93-101, 2014. PMID: 24892336. DOI: 10.1120/jacmp.v15i3.4641

20 Bradley JD, Paulus R, Komaki R, Masters G, Blumenschein G, Schild S, Bogart J, Hu C, Forster K, Magliocco A, Kavadi V, Garces YI, Narayan S, Iyengar P, Robinson C, Wynn RB, Koprowski C, Meng J, Beitler J, Gaur R, Curran W Jr and Choy $\mathrm{H}$ : Standard-dose versus high-dose conformal radiotherapy with concurrent and consolidation carboplatin plus paclitaxel with or without cetuximab for patients with stage IIIA or IIIB non-small- 
cell lung cancer (RTOG 0617): a randomized two-by-two factorial phase 3 study. Lancet Oncol 16(2): 187-199, 2015. PMID: 25601342. DOI: 10.1016/S1470-2045(14)71207-0

21 Chun SG, Hu C, Choy H, Komaki RU, Timmerman RD, Schild SE, Bogart JA, Dobelbower MC, Bosch W, Galvin JM, Kavadi VS, Narayan S, Iyengar P, Robinson CG, Wynn RB, Raben A, Augspurger ME, MacRae RM, Paulus R and Bradley JD: Impact of intensity-modulated radiation therapy technique for locally advance non-small-cell lung cancer: a secondary analysis of the NRG oncology RTOG 0617 randomized clinical trial. J Clin Oncol 35(1): 56-62, 2017. PMID: 28034064. DOI: 10.1200/JCO. 2016.69.1378

22 Reis CQM, Nicolucci P, Fortes SS and Sliva LP: Effects of heterogeneities in dose distributions under nonreference conditions: Monte Carlo simulation cs dose calculation algorithms. Med Dosim 44(1): 74-82, 2019. PMID: 29598926. DOI: $10.1016 /$ j.meddos.2018.02.009
23 Fogliata A, Nicolini G, Clivio A, Vanetti E and Cozzi L: Critical appraisal of Acuros XB and Anisotropic Analytic Algorithm dose calculation in advanced non-small-cell lung cancer treatments. Int J Radiat Oncol Biol Phys 83(5): 1587-1595, 2012. PMID: 22300575. DOI: 10.1016/j.ijrobp.2011.10.078

Received October 12, 2019

Revised October 30, 2019

Accepted November 5, 2019 